Max J. H. Worthington, ${ }^{\mathrm{a}, \mathrm{b}}$ Ismi Yusrina Muhti, ${ }^{\mathrm{b}}$ Maximilian Mann, ${ }^{\mathrm{a}, \mathrm{b}}$ Zhongfan Jia, ${ }^{\mathrm{a}, \mathrm{b}}$ Anthony D. Miller ${ }^{\mathrm{b}, *}$, and Justin M. Chalker ${ }^{\mathrm{a}, \mathrm{b} *}$

a) Institute for Nanoscale Science and Technology, College of Science and Engineering, Flinders University, Bedford Park, South Australia 5042, Australia

b) College of Science and Engineering, Flinders University, Bedford Park, South Australia 5042, Australia

To whom correspondence should be addressed:

tony.miller@flinders.edu.au

justin.chalker@flinders.edu.au

Keywords: mercury, polysulfide, remediation, sorbent, sulfur

\begin{abstract}
A polymer made from sulfur and limonene was used to coat silica gel and then evaluated as a mercury sorbent. A kinetic model of mercury uptake was established for a range of $\mathrm{pH}$ values and concentrations of sodium chloride. Mercury uptake was generally rapid from $\mathrm{pH}=3$ to $\mathrm{pH}=11$. At neutral $\mathrm{pH}$, the sorbent (500 mg with a 10:1 ratio of silica to polymer) could remove $90 \%$ of mercury within one minute from a $100 \mathrm{~mL}$ solution $5 \mathrm{ppm}$ in $\mathrm{HgCl}_{2}$ and 99\% over 5 minutes. It was found that sodium chloride, at concentrations comparable to seawater, dramatically reduced mercury uptake rates and capacity. It was also found that the spent sorbent was stable in acidic and neutral media, but degraded at $\mathrm{pH} 11$ which led to mercury leaching. These results help define the conditions under which the sorbent could be used, which is an important advance for using this material in remediation processes.
\end{abstract}

Statement of novelty: Previous studies of the featured mercury sorbent did not detail the scope and limitations at varying $\mathrm{pH}$ and salt concentrations, which are critical to know in remediation projects. This is the first study of the effects of $\mathrm{pH}$ and sodium chloride on the rate of mercury uptake by a polymer made from sulfur and limonene. Additionally, the first kinetic model of mercury uptake was established for this material. Finally, leaching experiments under different conditions were completed for the first time, establishing that the sorbent is stable at low and neutral $\mathrm{pH}$, but degrades at $\mathrm{pH} 11$ and releases bound mercury from the bulk sorbent.

Graphical abstract Renewable feedstock

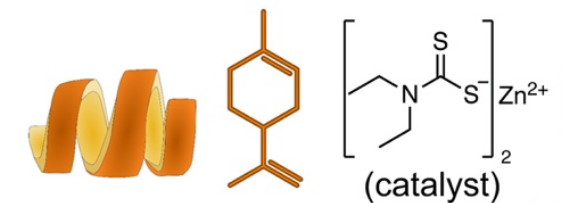

$140{ }^{\circ} \mathrm{C}$

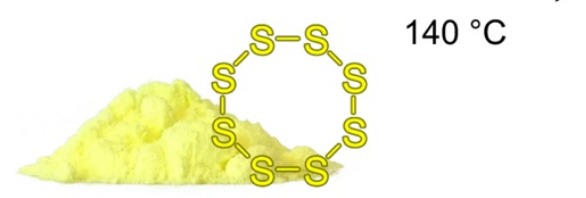

Surplus feedstock

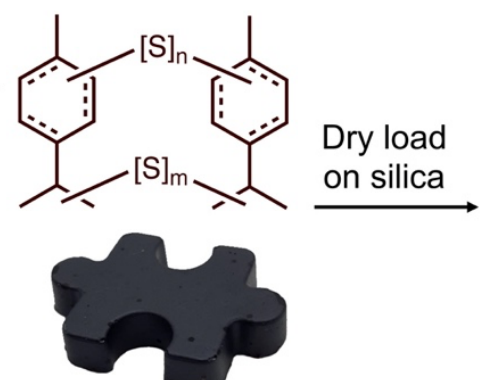

Solvent processible mercury sorbent

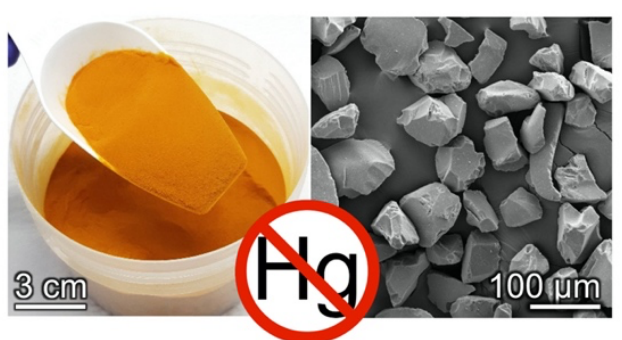

Free-flowing mercury sorbent Rapid mercury removal from water 


\subsection{INTRODUCTION}

Mercury is a toxic heavy metal encountered in a variety of industrial sectors such as coal combustion, oil and gas refining, and artisanal and small-scale gold mining. ${ }^{1,} 2$ Mercury pollution in aquatic systems is especially problematic, as it can contaminate the food supply through bioaccumulation in seafood, or lead to contaminated drinking water. ${ }^{3,4}$ Mercury remediation technologies for purifying water are therefore important, but this is challenging because of the high rate of mercury uptake required for filtration applications. High throughput is also important where large volumes of water need to be treated. To meet this need, a number of sorbent technologies have been evaluated including activated carbon, biochar, zeolites, polymers, metal covalent frameworks, and many other nanostructured or functionalised materials. ${ }^{5}$ Among the many classes of mercury sorbents, those functionalised with sulfur are of particular interest due to the strong affinity of sulfur for mercury. ${ }^{6} \mathrm{It}$ is perhaps not surprising that there have been many studies on sulfur-functionalised sorbents for mercury remediation. ${ }^{7}$,

8 Among these materials, those sorbents made directly from sulfur are of particular interest, due to the low cost and industrial surplus of elemental sulfur. ${ }^{7,9}$ Additionally, there has been a resurgence in methods to make polymers from sulfur, ${ }^{10-14}$ which has created many opportunities for using these low-cost and scalable polymers in mercury and heavy metal remediation. ${ }^{7}, 15-26$

In this study, we investigated one of these sulfur polymers made by direct copolymerisation of elemental sulfur with the renewable terpene limonene. Our lab first reported the synthesis and use of this material in mercury remediation in $2015,{ }^{27,} 28$ describing its advantageous features. For instance, both sulfur and limonene are low-cost, highly abundant feedstocks produced by the petroleum and citrus industries, respectively. The copolymer made from these building blocks_-referred to here as poly(S-r-limonene)—is a low molecular weight oligomer that is fully soluble in organic solvents. ${ }^{28}$ This solubility is useful, for instance, in using the polymer to coat surfaces. ${ }^{28,}{ }^{29}$ Additionally, at high concentrations of inorganic mercury, the polymer changes colour from red to yellow-a chromogenic response with potential use in mercury sensing or for filtration media that changes colour when it needs to be replaced. ${ }^{28}$ Building upon these foundational studies, the Hasell lab expanded access to this polymer and its utility in mercury remediation. For instance, the addition of an accelerator or catalyst in the synthesis allowed the material to be made at a lower temperature, which reduced by-product formation and increased the safety profile of the synthesis. ${ }^{29}$ Furthermore, Hasell also demonstrated that poly(S-r-limonene), when coated on a silica gel support, is effective at removing mercury from water. ${ }^{29}$ However, these studies were focused more on the poly(S-rlimonene) synthesis rather than mercury sorption, so there is a need to understand the detailed kinetics of mercury sorption and the scope of conditions under which it is effective. Herein, we report our findings on the first detailed kinetic analysis and modelling of this sorbent in both $\mathrm{Hg}^{2+}$ uptake and desorption. We also studied the influence of $\mathrm{pH}$ and sodium chloride concentrations on mercury sorption, which provided an increased understanding of the scope and limitations of this sorbent. These findings helped establish important guidelines for deploying this unique mercury sorbent in remediation.

\subsection{MATERIALS AND METHODS}

Additional experimental details, characterization data, and modelling details are provided in the Supporting Information.

\subsubsection{Synthesis of poly(S-r-limonene)}

D-Limonene $(20.00 \mathrm{~g}, 146.8 \mathrm{mmol})$ and zinc diethyldithiocarbamate $(2.00 \mathrm{~g}, 5.53 \mathrm{mmol})$ were added to a $100 \mathrm{~mL}$ round bottom flask and heated to $140{ }^{\circ} \mathrm{C}$ with stirring. Sulfur $(20.00 \mathrm{~g}, 625.0$ mmol) was added over 5 minutes to the reaction mixture. After the addition of sulfur, a heat gun was used to melt any sulfur adhering to the walls of the flask, returning the reagent to the reaction mixture. Over the course of 30 minutes, the reaction appeared to form one phase and changed from orange to dark brown. The reaction was continued for a period of 7 days. To isolate the product, the material was poured from the flask while hot, and then allowed to cool and solidify in a silicone mould. Yields were typically $>95 \%$ based on mass balance of the starting materials and product. 


\subsubsection{Coating silica gel with poly(S-r-limonene)}

The poly(S-r-limonene) material (13.89 g) was dissolved in $300 \mathrm{~mL}$ of dichloromethane and the solution was poured onto $138.9 \mathrm{~g}$ silica gel in a $1000 \mathrm{~mL}$ round bottom flask. The solvent was then removed by rotary evaporation $\left(40^{\circ} \mathrm{C}, 800 \mathrm{mbar}\right)$. In cases where the silica gel adhered together, additional dichloromethane was added and the dry loading repeated to ensure even coverage on the silica. Finally, the silica was dried under high vacuum to remove any remaining solvent. The coated silica was isolated as an orange, free-flowing powder (150 g).

\subsubsection{Preliminary assessment of mercury sorption}

An aqueous solution of $5 \mathrm{ppm} \mathrm{HgCl}_{2}(100 \mathrm{~mL})$ was added to a $250 \mathrm{~mL}$ round bottom flask containing $500 \mathrm{mg}$ of silica gel coated with poly(S-r-limonene). The sorbent was prepared as described above and contained a 10:1 mass ratio of silica to the poly(S-r-limonene) coating. The mixture was stirred and $1.00 \mathrm{~mL}$ samples of the solution were taken by pipette at 1, 2, 5, 10, 15, and 30 minutes. Any trace solids were removed by centrifugation, and the samples were diluted 10 -fold with $5 \% \mathrm{HCl}$ to stabilise mercury species for subsequent analysis. The experiment was repeated in triplicate. The experiment was also repeated with uncoated silica gel as a control. Mercury concentrations were then determined by cold vapour atomic absorption spectroscopic analysis (CVAAS).

\subsubsection{General protocol for kinetic analysis of mercury sorption}

Silica gel coated with poly(S-r-limonene) (500 mg, 10:1 silica:poly(S-r-limonene)) was added to $250 \mathrm{~mL}$ beaker and mixed with magnetic stirring. Next, an aqueous solution of $5 \mathrm{ppm} \mathrm{HgCl}_{2}$ $(100 \mathrm{~mL})$ was added. This solution was prepared at various $\mathrm{pH}$ values and sodium chloride concentrations, as described below (1.2.5 and 1.2.6). The solution was sampled over time by drawing $1.00 \mathrm{~mL}$ of the solution into a $3 \mathrm{~mL}$ syringe equipped with a syringe filter (nylon, 0.45 $\mu \mathrm{m})$. In this way, the solution was separated from the sorbent during sampling. Samples (1.00 $\mathrm{mL}$ ) were taken every 10 seconds for the first minute and then at $90,120,180$, and 300 seconds of total sorption time. The experiment was completed in triplicate. A control experiment was done in which $450 \mathrm{mg}$ of uncoated silica was used as the sorbent. All samples were diluted 10fold with $5 \% \mathrm{HCl}$ and then mercury concentrations were determined by CVAAS.

\subsubsection{Mercury sorption at varying $\mathrm{pH}$ values}

Aqueous solutions of $5 \mathrm{ppm} \mathrm{HgCl}_{2}$ were prepared at $\mathrm{pH}$ values of $3,5,9$, and 11 . The solution at $\mathrm{pH}=3$ contained $1 \mathrm{mM} \mathrm{HCl}$, the solution at $\mathrm{pH}=5$ contained $10 \mu \mathrm{m} \mathrm{HCl}$, the solution at $\mathrm{pH}=$ 9 contained $10 \mu \mathrm{m} \mathrm{NaOH}$, and the solution at $\mathrm{pH}=11$ contained $1 \mathrm{mM} \mathrm{NaOH}$. A 5 ppm solution of $\mathrm{HgCl}_{2}$ was also used without adjusting the $\mathrm{pH}$; this sample is referred to as the neutral sample $(\mathrm{pH}=6.99)$. The mercury solutions were added to the silica gel coated with poly(S-r-limonene) and sampled and analyzed by CVAAS according to the general protocol described in 1.2.4.

\subsubsection{Mercury sorption in the presence of $\mathrm{NaCl}$}

Aqueous solutions of $5 \mathrm{ppm} \mathrm{HgCl}_{2}$ and sodium chloride were prepared. The final concentration of sodium chloride was $6.85 \mathrm{mM}$ or $599 \mathrm{mM}$. These concentrations of sodium chloride are designed to mimic the salt concentrations in tap water and seawater, respectively. Both solutions were used in mercury sorption experiments following the general protocol for kinetic analysis described in 1.2.4.

\subsubsection{Mercury desorption assessment}

In a $500 \mathrm{~mL}$ beaker, a $20 \mathrm{ppm}$ solution of $\mathrm{HgCl}_{2}(200 \mathrm{~mL})$ was added along with silica gel coated with poly(S-r-limonene) (4.00 g). The sorbent was prepared as described above and contains a 10:1 mass ratio of silica to the poly(S-r-limonene) coating. The mixture was stirred for 10 minutes and then the sorbent was isolated by filtration and dried under vacuum before splitting up into $500 \mathrm{mg}$ portions. The sorbent samples, bound to mercury, were then added to $50 \mathrm{~mL}$ centrifuge tubes, followed by $50 \mathrm{~mL}$ of solutions of varying $\mathrm{pH}$ or sodium chloride concentrations ( $\mathrm{pH}$ of $3,5,7,9,11$, or aqueous solutions of $6.85 \mathrm{mM}$ or $599 \mathrm{mM} \mathrm{NaCl}$, prepared as described in 
the sorption experiments). The mixtures were rotated on an end-over-end mixer and sampled at 10 minutes, 3 hours, and then 1, 2, 8, 14, 21, and 28 days. All samples were diluted 10-fold with $5 \% \mathrm{HCl}$ and then mercury concentrations were determined by CVAAS.

\subsubsection{Mercury sorption modelling}

Two reaction models were used to analyse the kinetics of the $\mathrm{Hg}$ sorption experiments:

\subsubsection{Single reaction sorption model}

Let $\mathrm{X}$ denote an aqueous species and let $\mathrm{S}$ be an available adsorption site on the surface of the coating. Consider the conceptual reaction model

$$
\mathrm{X}[x]+\mathrm{S}[s] \rightarrow \mathrm{XS}[y]
$$

here XS denotes the adsorbed state. The symbols in square brackets [] denote the volume or surface concentrations, as appropriate, of the relevant species. In this model any reverse reaction has been assumed extremely slow and so it is neglected. Since this is modelled as an irreversible reaction, it will continue to completion until one of $\mathrm{X}$ or $\mathrm{S}$, or both are fully consumed. In what follows $\mathrm{X}$ can be thought of as $\mathrm{Hg}^{2+}$ or $\mathrm{HgCl}_{2}$.

A reaction rate equation for (1) can be written

$$
\frac{d y}{d t}=k_{1} \times s .
$$

If $x$ is expressed in molar units (M) then $k_{1}$ will have units of $\mathrm{M}^{-1} \mathrm{~s}^{-1}$

This rate equation is motivated by the following physical reasoning. The product term $x s$ is a measure of the likelihood of an entity of X and an entity of S coming into sufficiently close contact that the reaction (1) is possible, while $k_{1}$ is a proportionality factor that also expresses the likelihood that a close contact will actually result in the formation of the adsorbed complex XS. It is to be expected that $k_{1}$ will depend on a number of factors, in particular, the local chemical environment and temperature. The presence of other species, although they may not have any obvious direct involvement in the reaction, may give rise to various forms of interactions, for example crowding, shielding, attraction or repulsion, which can influence the likelihood of the reaction (1) occurring and so will affect $k_{1}$. Likewise, the presence of other nearby entities of $\mathrm{X}$ and $\mathrm{S}$ may also give rise to interactions and so influence whether a close contact leads to a reaction. So, $x$ and $s$ may affect the overall reaction rate not just through the product term $x s$, but also through $k_{1}$. However, it is assumed that $k_{1}$ does not change significantly during the course of the reactions considered here and consequently $k_{1}$ can be regarded as approximately constant. This constant may, however, have different values under different chemical conditions, such as widely different $\mathrm{pH}$ and $\mathrm{NaCl}$ concentrations as are investigated in the study.

\subsubsection{Two-reaction sorption model with competing reactions}

Let $\mathrm{W}$ be another aqueous species that potentially competes with $\mathrm{X}$ for adsorption sites $\mathrm{S}$. This gives the two-equation conceptual model

$$
\begin{aligned}
\mathrm{X}[x]+\mathrm{S}[s] & \rightarrow \mathrm{XS}[y] \\
\mathrm{W}[w]+\mathrm{S}[s] & \rightarrow \mathrm{WS}[y z]
\end{aligned}
$$

Again, reverse reactions have been neglected. These reactions will proceed to completion until either all of $\mathrm{S}$ is consumed, or all of both $\mathrm{X}$ and $\mathrm{W}$ are consumed. In the case that all of $\mathrm{S}$ is consumed then there will be some residual limiting presence of $\mathrm{X}$ or $\mathrm{W}$, or both. In what follows $\mathrm{W}$ will be either $\mathrm{NaOH}$ or $\mathrm{NaCl}$. 
Corresponding to (2) above is the set of two rate equations

$$
\begin{aligned}
& \frac{d y}{d t}=k_{1} x s \\
& \frac{d z}{d t}=k_{2} w s
\end{aligned}
$$

The same comments as above concerning the approximate constant value of $k_{1}$ apply here for $k_{1}$ and $k_{2}$ also. Supposing that both $x$ and $w$ are measured in units of $\mathrm{M}$, the units of both $k_{1}$ and $k_{2}$ are $\mathrm{M}^{-1} \mathrm{~s}^{-1}$.

\subsubsection{Desorption reaction model}

Any reverse reaction has been neglected in the above two sorption models. Over the longer term, however, desorption may take place to some degree. This can be accounted for by allowing the above reaction to be reversible. For the two-reaction model this would give

$$
\begin{gathered}
\mathrm{X}[x]+\mathrm{S}[s] \\
\mathrm{W}[w]+\mathrm{XS}[y] \\
\qquad \mathrm{WS}[y z]
\end{gathered}
$$

with corresponding rate equations

$$
\begin{aligned}
& \frac{d y}{d t}=k_{1} x s-k_{1 R} y \\
& \frac{d z}{d t}=k_{2} w s-k_{2 R} z
\end{aligned}
$$

Here the reverse rate coefficients $k_{1 R}$ and $k_{2 R}$ have units of $\mathrm{s}^{-1}$ or equivalent. Just as for $k_{1}$ and $k_{2}$, they will depend upon the chemical environment but are assumed approximately constant in any given situation.

\subsubsection{Stoichiometric ratio}

In the experimental protocols described above the starting volume concentrations of the aqueous species $\mathrm{X}$ and $\mathrm{W}$ are known. However, the starting surface concentrations and the effective surface area of the coating will not usually be known. Indeed, to some degree, these are idealised or conceptual quantities anyway. To overcome this difficulty the starting stoichiometric ratio

$$
r_{S}=\frac{\text { number of available (free) adsorption sites } \mathrm{S}}{\text { number of entities } \mathrm{X}}
$$

is introduced as another model parameter, in addition to the reaction rates.

\subsection{RESULTS AND DISCUSSION}

\subsubsection{Synthesis of polysulfide sorbent}

The direct reaction of limonene and elemental sulfur has been used to access polysulfide materials useful in mercury sorption. ${ }^{15,27,28}$ The starting materials are low cost, the synthesis is scalable to hundreds of grams, and at high concentrations of mercury the polysulfide changes colour-for potential use in mercury sensing. ${ }^{28}$ However, the original preparation of this has some shortcomings. For example, the reaction was carried out at $180{ }^{\circ} \mathrm{C}$ for several hours and distillation was required to remove low molecular weight byproducts such as $p$-cymene and malodorous thiols. ${ }^{28}$ Recently, it was discovered by Hasell and co-workers that the same material could be made at a lower temperature and with fewer byproducts through the use of an accelerator or catalyst such as zinc diethyldithiocarbamate. ${ }^{29,} 30$ This advance eliminated the need for the distillation step, as less limonene is oxidised to cymene and fewer thiols are formed. The latter feature means the product-referred to here as poly(S-r-limonene) - is not as malodorous as in the original synthesis. Hasell and co-workers also showed that poly(S-rlimonene), as a soluble oligomer, can be used to coat silica gel for mercury sorption. ${ }^{29}$ The focus of this report, however, was primarily on the use of accelerators and catalysts in the 
copolymerisation of sulfur with alkene-containing monomers, and not mercury sorption. Therefore, there is a need to characterise this sorbent in greater detail to understand its scope and limitations in mercury sorption. Accordingly, in this study we evaluated its use in mercury sorption and desorption at varying $\mathrm{pH}$ and salt concentrations, and we developed a model to account for the observed kinetics. Together, these results will help guide the deployment of this sorbent in mercury remediation.

The copolymerisation was run according to Hasell's protocol, reacting equal masses of sulfur and limonene directly in the presence of zinc diethyldithiocarbamate at $140{ }^{\circ} \mathrm{C}$ (Fig. 1 and Figs S1-S5). The accelerator was used at a loading of $5 \mathrm{wt} \%$ relative to the total mass of sulfur and limonene. Within 30 minutes the reaction appeared to form a single phase. After 24 hours, ${ }^{1} \mathrm{H}$ NMR analysis indicated complete consumption of the limonene alkenes (Fig. S6). To ensure that all sulfur was consumed, the reaction was continued for 7 days. The additional reaction time did not lead to detectable changes by ${ }^{1} \mathrm{H}$ NMR or gel permeation chromatography (GPC) but the viscosity did appear to increase somewhat over this time (Fig. S3). The molecular weight of the target oligomers was approximately $800 \mathrm{~g} / \mathrm{mol}$, based on GPC analysis (Figs S7-S12). This result was consistent across accelerator loadings of 0,1 , and $5 \mathrm{wt} \%$. The poly(S-r-limonene) with $5 \mathrm{wt} \%$ accelerator over 7 days was used for subsequent analysis and experiments. Simultaneous thermal analysis of this sample indicated a single mass loss starting at about $200{ }^{\circ} \mathrm{C}$ and no melting transition of elemental sulfur was observed, which is consistent with complete reaction of elemental sulfur (Figs S13-S14). Consistent with this result, powder X-ray diffraction analysis revealed an amorphous material, absent of crystalline $\mathrm{S}_{8}$ (Figs. S15-S16). Even after prolonged storage of this product (10 months), no crystalline sulfur was observed to form-a common phenomenon observed for this class of polysulfide materials. Differential scanning calorimetry indicated a glass transition temperature of $T_{\mathrm{g}}=8.6{ }^{\circ} \mathrm{C}$ (Fig. S14). Finally, combustible analysis was used to determine elemental composition, with $\mathrm{C}=39.8 \%, \mathrm{H}=4.9 \%$, $\mathrm{S}=53.5 \%$. For equal masses of limonene and sulfur, this is consistent with the expected values of $\mathrm{C}=44.08 \%, \mathrm{H}=5.92 \%, \mathrm{~S}=50.00 \%$, suggesting good atom economy and negligible loss of mass through volatilisation of monomers or formation of $\mathrm{H}_{2} \mathrm{~S}$. The elemental composition also remained consistent from day 3 to 7 of the synthesis (Fig. S18).

An advantage of the poly(S-r-limonene) oligomers is their solubility in organic solvents, facilitating use as a coating. To prepare poly(S-r-limonene) coated silica gel, $13.89 \mathrm{~g}$ of the oligomer was dissolved in $300 \mathrm{~mL}$ of dichloromethane and poured over $138.9 \mathrm{~g}$ of silica in a $1 \mathrm{~L}$ round bottom flask. The polymer was dry-loaded to the surface of the silica gel by rotary evaporation-rotating the sample while distilling the solvent at $40{ }^{\circ} \mathrm{C}$ and reduced pressure (800 mbar). The coated silica was then dried further under high vacuum to remove any remaining solvent. The product was a free-flowing orange powder (Figs 1 and S19). Examination under an electron microscope reveals no morphological differences between the coated and uncoated silica (Figs. S20-S21). Surface area of the coated silica was $273.40 \mathrm{~m}^{2} / \mathrm{g}$, as determined by Brunauer-Emmett-Teller (BET) analysis of nitrogen adsorption isotherms. The uncoated silica had a measured surface area of $444.16 \mathrm{~m}^{2} / \mathrm{g}$ (Fig. S22). The reduction in surface area for the coating sample is likely the result of the coating filling in some pores of the silica. Figure 1 summarizes the synthesis of poly(S-r-limonene) and shows the final sorbent after dry-loading the oligomer on silica. 
A. Limonene

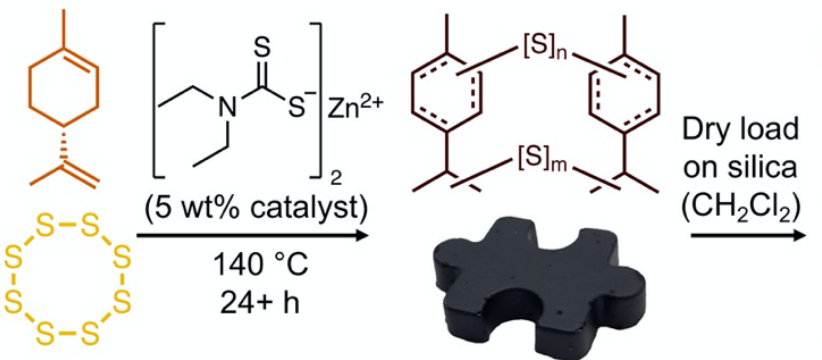

Sulfur

Solvent processible oligomer

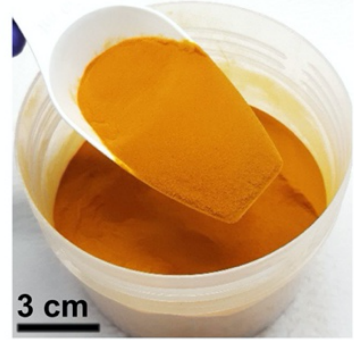

Free-flowing sorbent

B.

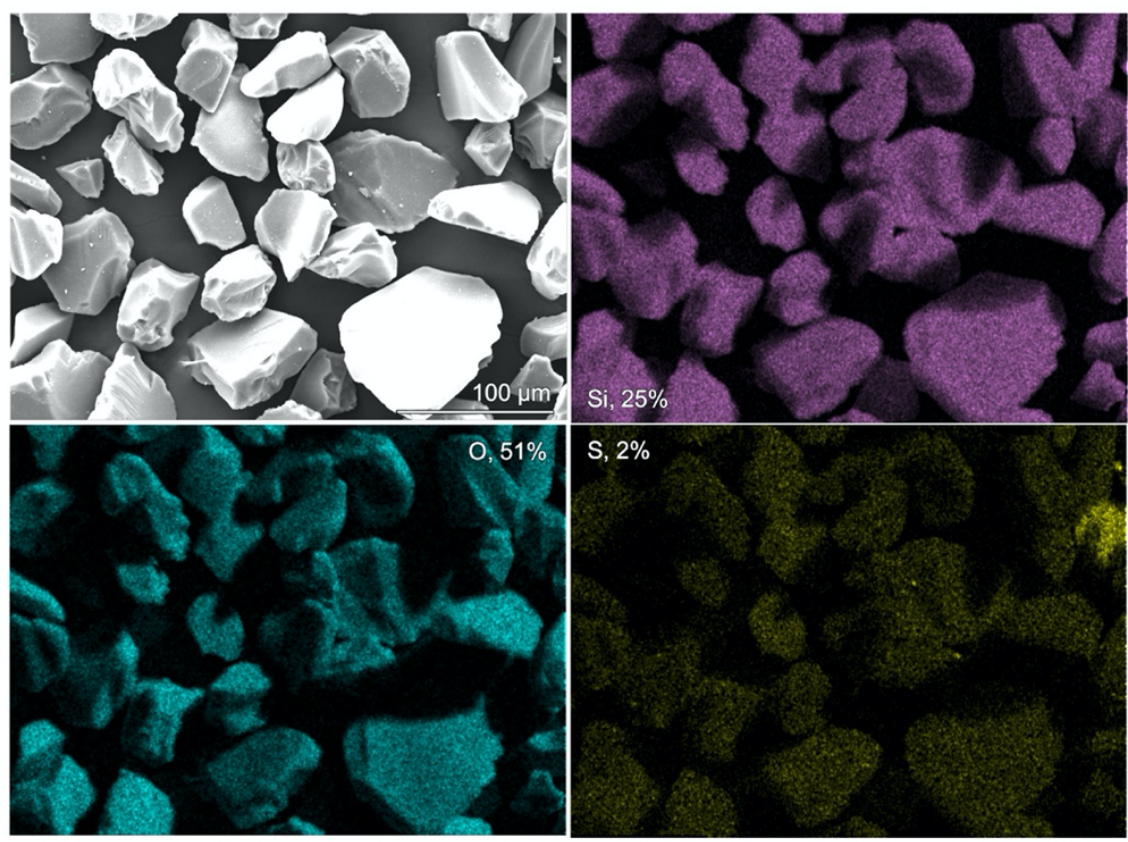

Figure 1: A. An oligomer was prepared by the direct reaction of equal masses of limonene and sulfur, catalyzed by zinc diethyldithiocarbamate. The oligomer, named poly(S-r-limonene), is fully soluble in organic solvents and can be coated onto silica gel using the dry loading method. B. Scanning electron microscopy (SEM) and energy-dispersive X-ray (EDX) imaging of the silica gel coated with poly(S-r-limonene). Additional characterization of the polymer and coated silica is provided in the Supporting Information.

\subsubsection{Sorption of $\mathrm{HgCl}_{2}$ in water}

In the initial evaluation of mercury sorption for the poly(S-r-limonene)-coated silica, 100 $\mathrm{mL}$ of an aqueous solution containing $5 \mathrm{ppm} \mathrm{HgCl}_{2}$ was added to $500 \mathrm{mg}$ of the sorbent. After 1 minute, a $1 \mathrm{~mL}$ sample of the solution was obtained and any trace solids were removed by centrifugation before analysis by CVAAS. Remarkably, $>90 \%$ of the mercury was removed from solution within this first minute (the average of triplicate experiments). No more than $20 \%$ of the mercury was removed by uncoated silica gel, indicating the key role of poly(S-r-limonene) in this rapid mercury uptake (Fig. S23). In order to monitor mercury sorption over smaller time intervals for kinetic analysis, a revised protocol was developed. Accordingly, $500 \mathrm{mg}$ of the poly(S-r-limonene)-coated silica was added to a $250 \mathrm{~mL}$ beaker followed by $100 \mathrm{~mL}$ of a $5 \mathrm{ppm}$ aqueous solution of $\mathrm{HgCl}_{2}$ with stirring. Samples were drawn up into a syringe equipped with a syringe filter to separate the sampled water from the sorbent during the sampling. Samples were obtained every 10 seconds for the first minute, and then after 90, 120, 180, and 300 seconds. Sorption was again rapid: Over $90 \%$ of mercury was sequestered within the first minute and over 98\% within 5 minutes. Kinetic modelling suggested that the maximum theoretical capacity of the sorbent for $\mathrm{Hg}^{2+}$ was $1 \mathrm{mg} / \mathrm{g}$ (equivalent to $11 \mathrm{mg} / \mathrm{g}$ of the poly(S-r-limonene coating) under these conditions (see more details below). This result was important in that the 
sorbent proved to be fast and effective at low concentrations of inorganic mercury, such as those often encountered in the environment. ${ }^{3,5}$

Next, similar sorption experiments were carried out with varying $\mathrm{pH}$ and $\mathrm{NaCl}$ concentrations. These experiments were designed to help define under what conditions the sorbent is effective in aqueous media. The $\mathrm{pH}$ was adjusted to values of $3,5,9$, and 11 (all $5 \mathrm{ppm}$ in $\left.\mathrm{HgCl}_{2}\right)$ and compared to the unmodified $5 \mathrm{ppm}$ solution of $\mathrm{HgCl}_{2}$ in water $(\mathrm{pH}=6.99)$. For the effects of sodium chloride on mercury sorption, concentrations of $6.85 \mathrm{mM}$ and $599 \mathrm{mM} \mathrm{NaCl}$ were evaluated-designed to mimic the salt concentrations in tap water and seawater, respectively. Sampling was carried out as describe above, with mercury concentration monitored over 300 seconds by CVAAS. The results are plotted in Figure 2. Key findings were that mercury sorption was most effective at $\mathrm{pH}=7$ or lower. However, mercury sorption slowed significantly at higher $\mathrm{pH}$ and bound less total mercury (see kinetic section below for additional details). The most dramatic change in sorption was at high concentrations of $\mathrm{NaCl}$, with 599 $\mathrm{mM} \mathrm{NaCl}$ significantly inhibiting mercury sorption. At this concentration of $\mathrm{NaCl}$, only $30 \%$ of mercury was removed by the poly(S-r-limonene)-coated silica over 5 minutes, while $86 \%$ of mercury was removed when the concentration of $\mathrm{NaCl}$ was $6.85 \mathrm{mM}$. When no exogenous $\mathrm{NaCl}$ was added, $99 \%$ of the mercury was removed by $500 \mathrm{mg}$ of the sorbent from a $100 \mathrm{~mL}$ solution of $5 \mathrm{ppm}$ mercury. These results are summarized in Figure 2. Additional data is provided in Figures S24-S29.
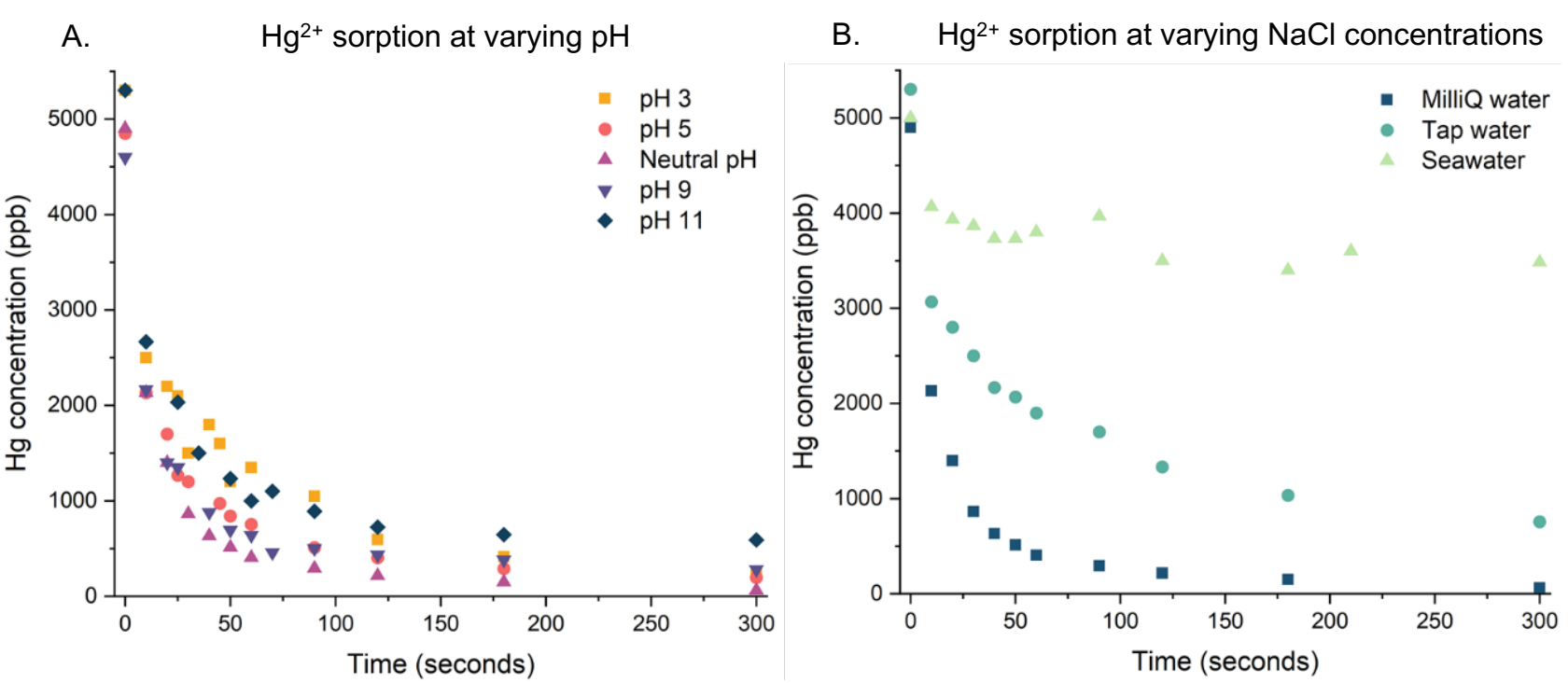

Figure 2: Silica coated with poly(S-r-limonene) (10:1 silica to polymer) was used in mercury sorption experiments at varying $\mathrm{pH}$ and sodium chloride concentrations. A. $500 \mathrm{mg}$ of the sorbent was added to a $5000 \mathrm{ppb}$ solution of $\mathrm{HgCl}_{2}$ at $\mathrm{pH}=3,5,7,9$, and 11 . The sorption was slower at higher pH. B. $500 \mathrm{mg}$ of the sorbent was added to a $5000 \mathrm{ppb}$ solution of $\mathrm{HgCl}_{2}$ in MilliQ purified water (no added $\mathrm{NaCl})$, simulated tap water $(6.85 \mathrm{mM} \mathrm{NaCl})$, and simulated seawater $(599 \mathrm{mM} \mathrm{NaCl})$. The exogenous sodium chloride slowed mercury sorption.

\subsubsection{Kinetic analysis of $\mathrm{HgCl}_{2}$ sorption}

A graphical fitting procedure was used to match the experimental results to the predictions of the kinetic models. The various model parameters $\left(k_{1}, k_{2}, r_{S}\right)$ were varied until the predictions appeared to provide the best visual fit to the log-log representation of the experimental data (Fig. 3 and Figs S30-S33). There were two main reasons for using the log-log representation in this analysis.

1. A log-log scale is a non-linear scale that expands small values of time (horizontal axis) and concentration (vertical axis) relative to larger values. This focuses attention on the time and concentration regions that are most important: early times when there is a rapid initial decrease in concentration, and low values of concentration so as to better see the long term trend in concentration. 
In most cases, varying $k_{1}$ from its best fitted value by $\pm 15 \%$ or more produced a noticeably poorer fit. So $\pm 15 \%$ can be thought of as an informal confidence interval for the parameter estimates. A summary of the results is given in the Table 1. A few explanatory notes are listed below:

1. For the sample in which the $\mathrm{pH}$ was not adjusted (neutral sample, $\mathrm{pH}=6.99$ ), $r_{S}$ was taken to be a model parameter to be estimated. Its estimated value was found to be $r_{S} \approx$ 1, implying that the sorbent and aqueous $\mathrm{HgCl}_{2}$ are close to stoichiometric balance (Fig. S30). Changing $r_{S}$ by as little as $\pm 2.5 \%$, produced noticeable deviations from the experimental data. Since all other experimental cases used the same amounts of $\mathrm{HgCl}_{2}$ and sorbent, it was supposed that $r_{S}$ would be unchanged, and it was given the fixed value $r_{S}=1$ for these cases also.

2. For the neutral case $(\mathrm{pH}=6.99)$ and the acidic cases $(\mathrm{pH}=3$ and $\mathrm{pH}=5)$ the single reaction model was adequate to fit the experimental data well (Fig. S31). All these cases show a continuing decrease in $\mathrm{Hg}$ concentration with time over the sampling period. Indeed, the log-log plots asymptote to a slope of -1 , indicating a $1 / t$ asymptotic behaviour (in non-logarithmic units). The physical interpretation of these results is that ultimately all $\mathrm{X}$ will be adsorbed and all adsorption sites $\mathrm{S}$ will be utilised.

3. For the basic cases $(\mathrm{pH}=9$ and $\mathrm{pH}=11)$ the two-reaction model was needed to fit the apparent steady state that was approached. Here the competing species W was taken to be $\mathrm{NaOH}$. The physical interpretation of these results is that ultimately all adsorption sites $\mathrm{S}$ will be utilised, some by $\mathrm{X}$, and the remainder by W. The ratio of the amounts of $\mathrm{X}$ and $\mathrm{W}$ adsorbed depends on the ratio $k_{1} / k_{2}$ as well as the starting stoichiometric ratios $r_{S}$ and $r_{W}$. Further details can be found in the Supplementary Material (Fig. S32).

4. For the tap and seawater cases, the two-reaction model was again needed to fit the apparent steady state that was approached (Fig. S33). Here the competing species W was taken to be $\mathrm{NaCl}$. The physical interpretation is similar to that for the base cases but with $\mathrm{NaCl}$ as the competing species.

5. For the seawater case there was only a small reduction in $\mathrm{Hg}$ concentration during the 5 minutes of the experiment as the apparent steady state was approached. Because of this the model parameters, in particular $k_{1}$, could not be estimated with great accuracy. An informal confidence range for $k_{1}$ has been given in Table 1 . 

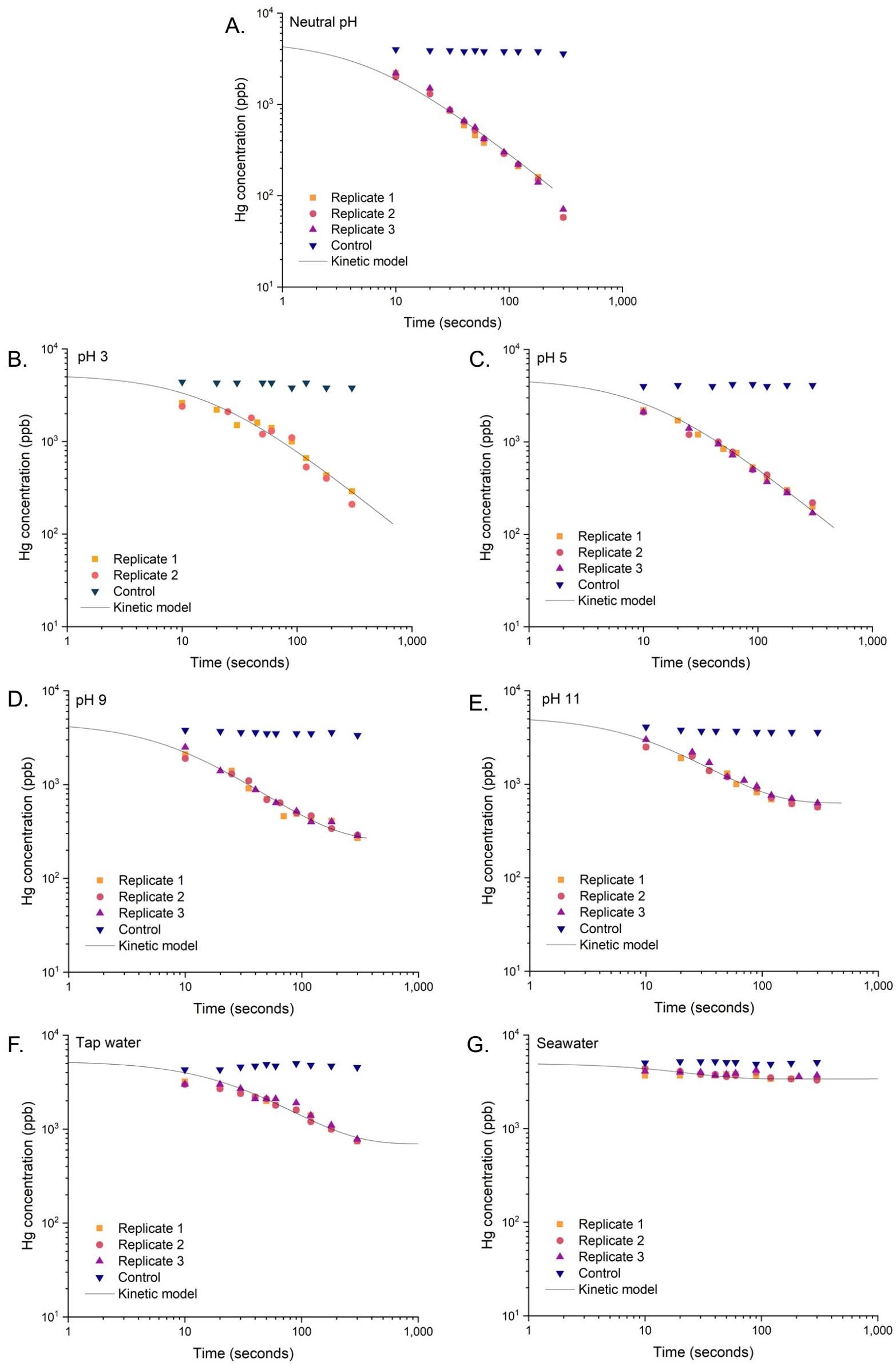

Figure 3: Kinetic models fit to mercury sorption data on log-log scales. A. Neutral pH, no added $\mathrm{NaCl}$. B. pH 3. C. pH 5. D. pH 9. E. pH 11. F. Simulated tap water (6.85 mM NaCl) G. Simulated 
Table 1: Estimated values of the model parameters $k_{1}$ and $k_{2}$ based on fitting to experimental data. Unless specified otherwise, varying $k_{1}$ by more than $\pm 15 \%$ produced predictions that are noticeably different from the experimental results. Based on the $\mathrm{pH}=7$ results, $r_{S}$ was estimated to be $r_{S}=1 \pm 0.025$, and this value was used for all other cases

422

423

424

425

426

427

428

429

430

431

432

433

434

435

\begin{tabular}{lllll} 
Case & $\boldsymbol{k}_{\mathbf{1}}\left[\mathbf{M}^{-1} \mathbf{s}^{-1}\right]$ & $\boldsymbol{k}_{\mathbf{2}}\left[\mathbf{M}^{-1} \mathbf{s}^{-1}\right]$ & $\boldsymbol{k}_{\mathbf{2}} / \boldsymbol{k}_{\mathbf{1}}$ & Comment \\
\hline pH 3 & $2.2 \times 10^{3}$ & & & single reaction model \\
pH 5 & $3.6 \times 10^{3}$ & & & single reaction model \\
pH 7 & $6.7 \times 10^{3}$ & & & single reaction model, $r_{S}=1$ \\
pH 9 & $4.9 \times 10^{3}$ & $2.2 \times 10^{2}$ & $4.6 \times 10^{-2}$ & \\
pH 11 & $3.2 \times 10^{3}$ & 4.3 & $1.4 \times 10^{-3}$ & \\
Tap water & $1.3 \times 10^{3}$ & 0.32 & $2.5 \times 10^{-4}$ & \\
6.8 $10^{-3} \mathrm{M} \mathrm{NaCl}$ & & & & \\
Saltwater & $6.7 \times 10^{2}$ & $4.9 \times 10^{-2}$ & $7.3 \times 10^{-5}$ & $k_{1} \approx 4 \times 10^{2}-1 \times 10^{3}$ \\
0.60 M NaCl & & & &
\end{tabular}

\section{Variation of $\boldsymbol{k}_{1}$ across experimental conditions}

It is instructive to plot $1 / k_{1}$ against $\mathrm{pH}$ or the log of the chloride ion concentration (as appropriate for the case). This is shown in Figure 4. As all cases were prepared from $\mathrm{HgCl}_{2}$, there was always a background $\mathrm{Cl}^{-}$concentration arising from the $5 \mathrm{ppm} \mathrm{HgCl}_{2}$. For this reason, the $\mathrm{pH}=7$ case has been treated as a (low level) reference for the acid, base and chloride cases. In the figure each of the acid (blue), base (red) and $\mathrm{NaCl}$ (black) segments are approximately straight lines over the ranges of concentration considered. This observation needs to be treated with caution as it is based on only three data points for each segment, and as noted above, there is considerable uncertainty in the estimates of $k_{1}$. Moreover, $1 / k_{1}$ must have a strictly positive limit as the concentrations of $\mathrm{H}^{+}, \mathrm{HO}^{-}$or $\mathrm{Cl}^{-}$tend to 0 . Nonetheless, these plots give some indication that the presence in high concentrations of $\mathrm{H}^{+}, \mathrm{HO}^{-}$or $\mathrm{Cl}$ - decreases $k_{1}$ (increases $\left.1 / k_{1}\right)$ in a systematic way.

$1 / \mathrm{k} 1 \mathrm{vs} \mathrm{pH}$ and chloride concentration

Chloride concentration (M)

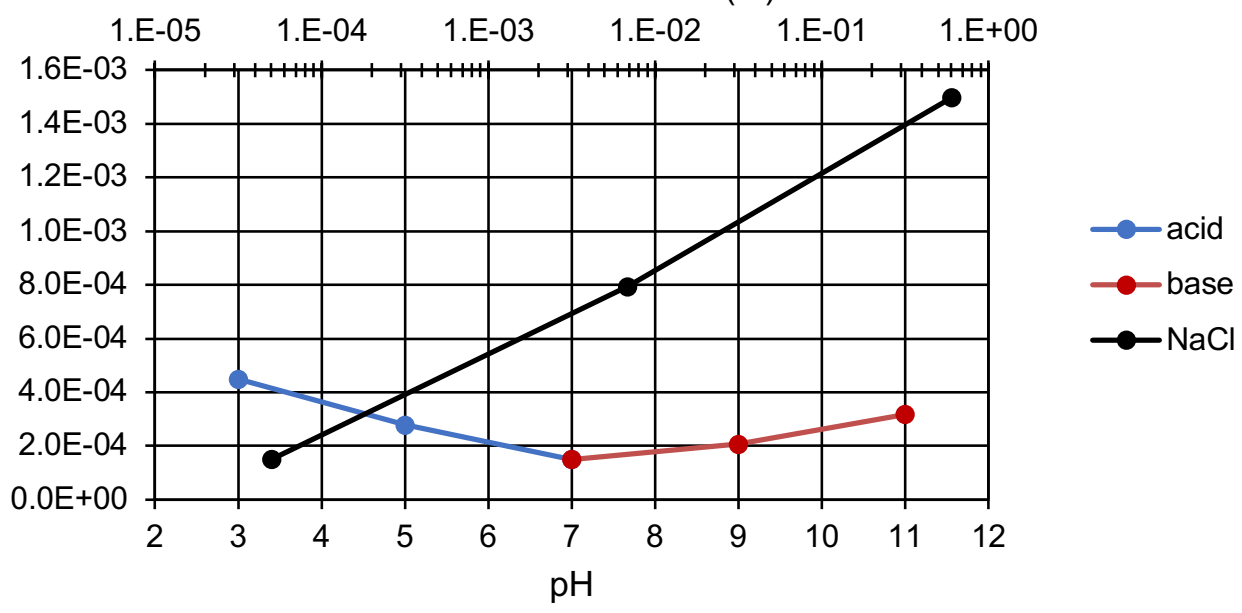

Figure 4: Plot of $1 / k_{1}$ vs $\mathrm{pH}$ (for acid and base cases) and chloride concentration (for $\mathrm{NaCl}$ cases). There are different segments for each case. The estimated $k_{1}$ value for the $\mathrm{pH}=7$ experiment is plotted as a (reference) data point on both the acid and base segments, and also as the leftmost data point on the $\mathrm{NaCl}$ segment. 


\subsubsection{Influence of pH on mercury sorption kinetics}

As seen in Table 1 and Figure 4, the poly(S-r-limonene) sorbent is fastest at neutral or nearneutral $\mathrm{pH}$, with reduced rates of mercury uptake below or above $\mathrm{pH} 7$. For example, within 5 minutes, $500 \mathrm{mg}$ sorbent captured $95,96,94$ and $90 \%$ of mercury from solutions at $\mathrm{pH} \mathrm{3,5,9}$ and 11 , respectively. In contrast, the sorbent removed $99 \%$ of the mercury in the same 5 minute period in the neutral sample under otherwise identical conditions. The $\mathrm{pH}$ might alter the mercury speciation, which could account for these differences in rates of mercury uptake. Note that for neutral and low $\mathrm{pH}$ the single reaction model fits the experimental data well, suggesting that the availability of sorption sites is not significantly affected by these $\mathrm{pH}$ values. This is also shown by Figure 3 where there is no apparent flattening of the curves for cases A, B and C. This contrasts with the high $\mathrm{pH}$ cases where a two-reaction model with a competing reaction was required. For high $\mathrm{pH}$, hydroxide might potentially break S-S bonds in the polysulfide which could hinder both the measured rate of mercury sorption in addition to the capacity of the sorbent, which is reflected in the flattening of the model curve in Figures 3D and 3E. Indeed prolonged exposure to high $\mathrm{pH}$ resulted in degradation of the polymer coating (see desorption experiments for additional discussion of this observation).

\subsubsection{Influence of sodium chloride on mercury sorption kinetics}

Sodium chloride clearly interfered with the mercury sorption of poly(S-r-limonene)-an

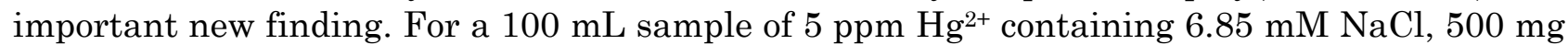
of the poly(S-r-limonene)-coated silica gel only removed $86 \%$ of the mercury after 5 minutes. For a $100 \mathrm{~mL}$ sample of $5 \mathrm{ppm} \mathrm{Hg}^{2+}$ containing $599 \mathrm{mM} \mathrm{NaCl}, 500 \mathrm{mg}$ of the poly(S-r-limonene)coated silica gel only removed $30 \%$ of the mercury after 5 minutes. In contrast, with no exogenous $\mathrm{NaCl}$ added in an otherwise identical experiment, 99\% of the mercury was removed. The origin of the inhibition can be multi-faceted, but the models in Figure 3F and 3G clearly indicate that the $\mathrm{NaCl}$ reduces both the rate of mercury uptake and the amount of mercury that can be bound. The latter point is indicative of $\mathrm{NaCl}$ competing for or otherwise blocking binding sites. Indeed, $\mathrm{NaCl}$ particles could be detected directly on the sorbent. For instance, incubating the poly(S-r-limonene)-coated silica in water with $599 \mathrm{mM} \mathrm{NaCl}$ for one month resulted in substantial amounts of sodium chloride on the surface of the polymer, even after isolating by filtration and washing with water. It might also be important to consider chloride as a ligand for $\mathrm{Hg}^{2+}$, which may compete with sulfur in mercury binding. In any case, it is clear that the sorbent's effectiveness is reduced with increasing $\mathrm{NaCl}$. This suggests that its use in remediation of seawater may not be practical or require more sorbent than for samples with lower levels of $\mathrm{NaCl}$, as discussed next.

To test if a higher concentration of sorbent can achieve more complete mercury binding in the presence of sodium chloride, 9 times the mass of sorbent was used in an identical sorption experiment. The kinetic model was used to predict that this amount of sorbent would provide sufficient binding sites to overcome the competing processes with $\mathrm{NaCl}$. The qualitative concept here was that solely by a mass action effect, adding more sorbent would speed up both the desired mercury sorption and the undesired competing reaction. However, due to the relative rates of these reactions, $k_{1} / k_{2}$, the sorption reaction is now able to proceed further before a steady state is achieved. Accordingly, $4.5 \mathrm{~g}$ poly(S-r-limonene) coated silica was mixed with 100 $\mathrm{mL} 5 \mathrm{ppm} \mathrm{Hg}^{2+}$ containing $599 \mathrm{mM} \mathrm{NaCl}$ for 5 minutes with regular sampling. Within the first 10 seconds the sorbent removed $83 \%$ of the mercury present-a greatly improved initial uptake than observed in the original experiment with 9-times less sorbent. Over 5 minutes, $91 \%$ of the mercury was removed (Fig S36). This experiment shows that additional sorbent can overcome the detrimental effect of sodium chloride on mercury binding performance as predicted from the kinetic model.

\subsection{Desorption of mercury from sorbent}

An important factor to consider in remediation is the stability of the spent sorbent. For instance, it is important that the mercury does not leach during the transport and storage of the spent 
sorbent. To evaluate desorption of mercury from the spent sorbent, $4.00 \mathrm{~g}$ of the poly(S-rlimonene)-coated silica was added to a $200 \mathrm{~mL}$ aqueous solution of $\mathrm{HgCl}_{2}(20 \mathrm{ppm})$. The mixture was stirred for 10 minutes and then the sorbent was isolated by filtration and dried under vacuum. Based on the mercury remaining in the water (as determined by CVAAS), the spent sorbent bound $1.03 \mathrm{mg} \mathrm{Hg}{ }^{2+}$ per gram of sorbent. To evaluate leaching, $500 \mathrm{mg}$ portions of the spent sorbent was added to separate $50 \mathrm{~mL}$ aqueous solutions of varying $\mathrm{pH}$ and sodium chloride concentrations $(\mathrm{pH}=3,5,7,9,11$ and $6.85 \mathrm{mM}$ or $599 \mathrm{mM} \mathrm{NaCl})$. These seven samples were monitored over 28 days to determine how much mercury leached into the water. Minimal leaching was observed for all samples except for the sample at $\mathrm{pH}=11$ (Figs. 5A and S37). After 28 days, approximately $3 \%$ of the bound mercury had leached into the basic solution at $\mathrm{pH}=11$. All other samples had mercury levels at or below the limits of detection by CVAAS, indicating leaching was not significant for $\mathrm{pH}=2,5,7$, or 9. Leaching was also not significant for the samples with added sodium chloride. SEM analysis of the sorbent did reveal that the high $\mathrm{pH}$ degrades the polymer coating (Figs 5B and S37). This could lead to the release of mercury, perhaps bound to suspended polymer particles (or the products of polymer degradation). Further study is required to determine the mechanism and speciation of the mercury leaching at elevated $\mathrm{pH}$. However, minimal leaching was observed for all other samples suggesting that the mercury remains strongly bound to the sorbent even in highly acidic media or brine.

A. Mercury leaching from spent sorbent

B. Degraded sorbent coating at $\mathrm{pH}=11$

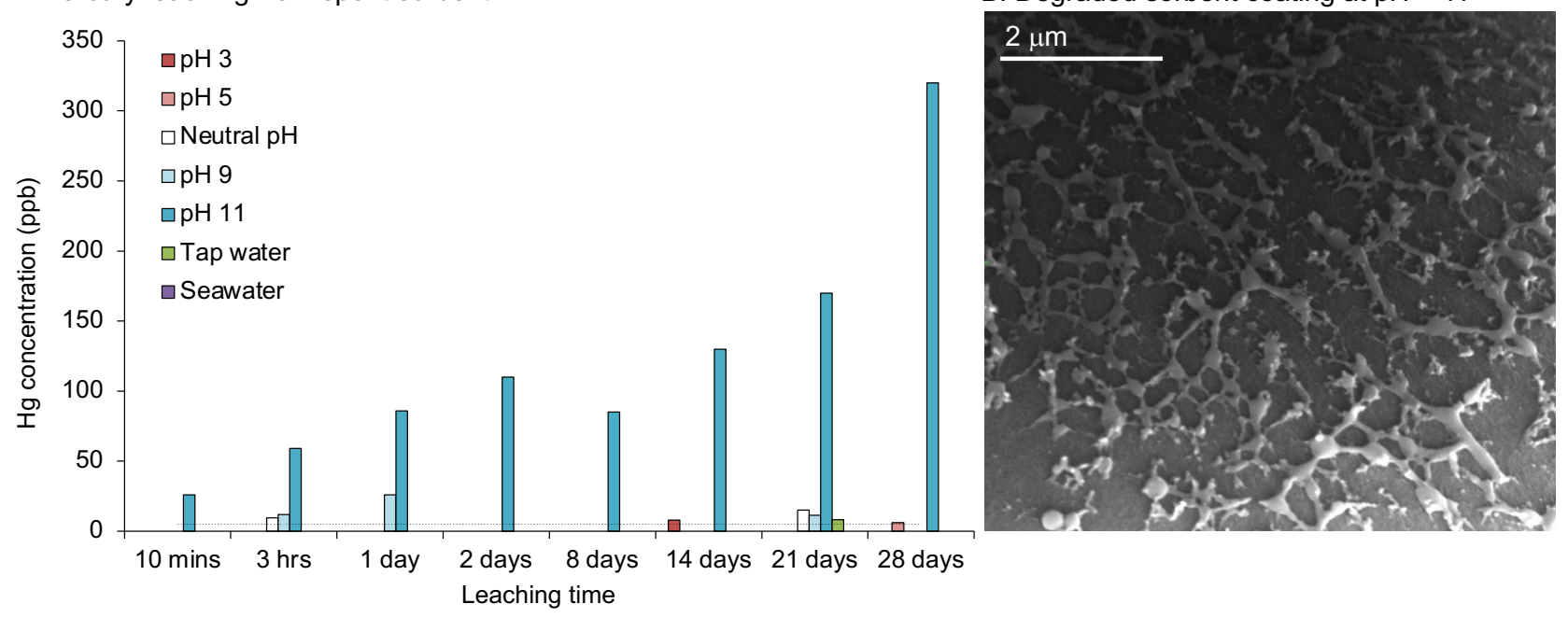

Figure 5: A. The spent sorbent (saturated at $1 \mathrm{mg} \mathrm{HgCl} 2$ per gram sorbent) was subjected to leaching experiments at varying $\mathrm{pH}$ and sodium chloride concentrations. A mass of $500 \mathrm{mg}$ of the spent sorbent was added to $50 \mathrm{~mL}$ of the aqueous solution for these leaching experiments. The dotted line represents the limit of detection of $5 \mathrm{ppb}$. Only at $\mathrm{pH} 11$ was significant leaching observed. After 28 days, this leaching still only corresponds to $3 \%$ of the bound mercury. B. SEM micrograph of the sorbent after the leaching experiment at $\mathrm{pH} 11$. The coating appears to have been degraded at the high $\mathrm{pH}$, as the surface was no longer smooth as seen in Figs 1 and in the other leaching experiments (Figs. S38-S47).

\subsection{Conclusions}

Poly(S-r-limonene)-coated silica was evaluated as a mercury sorbent over a range of $\mathrm{pH}$ values and also in the presence of sodium chloride. The sorbent rapidly removed $\mathrm{HgCl}_{2}$ from water at or near neutral $\mathrm{pH}$. Slightly reduced rates of uptake were observed at both low and high $\mathrm{pH}$, but the sorbent was still effective across this wide $\mathrm{pH}$ range. One-reaction or two-reaction kinetic models were fitted to the experimental sorption results. These modes suggest that under low (acid) and neutral $\mathrm{pH}$ conditions, mercury sorption is a single reaction process which will ultimately proceed to completion until one or both of the mercury or the sorbent is consumed. In contrast, for basic $\mathrm{pH}$ and in the presence of $\mathrm{NaCl}$, the models suggest that there are significant competing reactions whereby some of the sorbent becomes unavailable for mercury binding. It was also discovered that sodium chloride severely inhibits mercury binding, which could limit the use of the sorbent in salt water systems. The spent sorbent was found to be stable 
and did not leach significant mercury from $\mathrm{pH}=3$ to $\mathrm{pH}=9$. Aqueous sodium chloride also did not lead to leaching. However, at $\mathrm{pH}=11$ the coating degraded and mercury was released into the solution, possibly bound to suspended polymer particles or other polymer degradation products. Together, these results suggest that the sorbent is most effective at low and neutral $\mathrm{pH}$ and that elevated $\mathrm{pH}$ can lead to polymer degradation. This assessment of the scope and limitations of this sorbent will help define the conditions for which it is most effective in the field. While this study focussed on inorganic mercury, future studies will be carried out that evaluate the sorbent on a broader range of mercury species and field samples.

\section{Author contributions}

Max J. H. Worthington: Data curation, Formal analysis, Investigation, Methodology, Visualization, Writing - original draft, Writing - review and editing

Maximilian Mann: Data curation, Formal analysis, Methodology, Investigation, Writing review and editing

Ismi Yusrina Muhti: Data curation, Formal analysis, Methodology, Investigation, Writing review and editing

Zhongfan Jia: Formal analysis, Investigation, Supervision, Writing - review and editing.

Anthony D. Miller: Conceptualization, Data curation, Formal analysis, Methodology, Project administration, supervision, Visualization, Writing - original draft, Writing - review and editing

Justin M. Chalker: Conceptualization, Formal analysis, Funding acquisition, Methodology, Project administration, Resources, Supervision, Writing - original draft, Writing - review and editing

\section{Acknowledgements}

This work was supported by the Australian Research Council (DP200100090). The authors also acknowledge the support of Flinders Microscopy and Microanalysis for access to polymer characterisation facilities.

\section{References}

1. Global Mercury Assessment 2018, UN Environment Programme, Chemicals and Health Branch, Geneva, Switzerland.

2. $\quad$ L. J. Esdaile and J. M. Chalker, Chem. Eur. J., 2018, 24, 6905-6916.

3. Q. Wang, D. Kim, D. D. Dionysiou, G. A. Sorial and D. Timberlake, Environ. Pollut., 2004, 131, 323-336.

4. P. B. Tchounwou, W. K. Ayensu, N. Ninashvili and D. Sutton, Eviron. Toxicol., 2003, 18, 149-175.

5. L. Wang, D. Hou, Y. Cao, Y. S. Ok, F. M. G. Tack, J. Rinklebe and D. O'Connor, Environ. Int., 2020, 134, 105281.

6. R. G. Pearson, J. Am. Chem. Soc., 1963, 85, 3533-3539.

7. J. M. Chalker, M. Mann, M. J. H. Worthington and L. J. Esdaile, Org. Mater., 2021, 3, 362-373.

8. M. Mann, X. Luo, A. D. Tikoalu, C. T. Gibson, Y. Yin, R. Al-Attabi, G. G. Andersson, C. L. Rason, L. C. Henderson, A. Pring, T. Hasell and J. M. Chalker, Chem. Commun., 2021, 57, 6296-6299.

9. T. Tian, R. Hu and B. Z. Tang, J. Am. Chem. Soc., 2018, 140, 6156-6163.

10. W. J. Chung, J. J. Griebel, E. T. Kim, H. Yoon, A. G. Simmonds, H. J. Ji, P. T. Dirlam, R. S. Glass, J. J. Wie, N. A. Nguyen, B. W. Guralnick, J. Park, A. Somogyi, P. Theato, M. E. Mackay, Y.-E. Sung, K. Char and J. Pyun, Nat. Chem., 2013, 5, 518-524.

11. Y. Zhang, R. S. Glass, K. Char and J. Pyun, Polym. Chem., 2019, 10, 4078-4105.

12. M. J. H. Worthington, R. L. Kucera and J. M. Chalker, Green Chem., 2017, 19, 27482761.

13. J. M. Chalker, M. J. H. Worthington, N. A. Lundquist and L. J. Esdaile, Top. Curr. Chem., 2019, 377, 16. 
14. T. Lee, P. T. Dirlam, J. T. Njardarson, R. S. Glass and J. Pyun, J. Am. Chem. Soc., $2022, \mathbf{1 4 4}, 5-22$.

15. T. Hasell, D. J. Parker, H. A. Jones, T. McAllister and S. M. Howdle, Chem. Commun., 2016, 52, 5383-5386.

16. M. W. Thielke, L. A. Bultema, D. D. Brauer, B. Richter, M. Fischer and P. Theato, Polymers, 2016, 8, 266.

17. J. M. Scheiger, C. Direksilp, P. Falkenstein, A. Welle, M. Koenig, S. Heissler, J. Matysik, P. A. Levkin and P. Theato, Angew. Chem. Int. Ed., 2020, 59, 18639-18645.

18. D. J. Parker, H. A. Jones, S. Petcher, L. Cervini, J. M. Griffin, R. Akhtar and T. Hasell, J. Mater. Chem. A, 2017, 5, 11682-11692.

19. L. A. Limjuco, G. M. Nisola, K. J. Parohinog, K. N. G. Valdehuesa, S.-P. Lee, H. Kim and W.-J. Chung, Chem. Eng. J., 2019, 378, 122216.

20. S. Akay, B. Kayan, D. Kalderis, M. Arslan, Y. Yagci and B. Kiskan, J. Appl. Polym. Sci., 2017, 134, 45306.

21. A. M. Abraham, S. V. Kumar and S. M. Alhassan, Chem. Eng. J. , 2018, 332, 1-7.

22. V. S. Wadi, H. Mittal, E. Fosso-Kankeu, K. K. Jena and S. M. Alhassan, Colloids Surf., $A, 2020,606,125333$.

23. J. Lee, S. Lee, J. Kim, Z. Hanif, S. Han, S. Hong and M.-H. Yoon, Bull. Korean Chem. Soc., 2018, 39, 84-89.

24. L. A. Limjuco, H. T. Fissaha, H. Kim, G. M. Nisola and W.-J. Chung, ACS Appl. Polym. Mater., 2020, 2, 4677-4689.

25. M. Mann, B. Zhang, S. J. Tonkin, C. T. Gibson, Z. Jia, T. Hasell and J. M. Chalker, Polym. Chem., 2022, in press. DOI: doi.org/10.1039/D1PY01416A.

26. M. L. Eder, C. B. Call and C. L. Jenkins, ACS Appl. Polym. Mater., 2022, in press, DOI: 10.1021/acsapm.1c01536.

27. M. P. Crockett, A. M. Evans, M. J. H. Worthington, J. M. Chalker, Sulfur-Limonene Polysulfide. US Patent 10,590,012, 2020. Priority date 13 Oct 2015.

28. M. P. Crockett, A. M. Evans, M. J. H. Worthington, I. S. Albuquerque, A. D. Slattery, C. T. Gibson, J. A. Campbell, D. A. Lewis, G. J. L. Bernardes and J. M. Chalker, Angew. Chem. Int. Ed., 2016, 55, 1714-1718.

29. X. Wu, J. A. Smith, S. Petcher, B. Zhang, D. J. Parker, J. M. Griffin and T. Hasell, Nat. Commun., 2019, 10, 647.

30. L. James Dodd, Ö. Omar, X. Wu and T. Hasell, ACS Catal., 2021, 11, 4441-4455. 